\title{
Seven problems on hypohamiltonian and almost hypohamiltonian graphs
}

\author{
Carol T. Zamfirescu*
}

2010 Mathematics Subject Classification: 05C10, 05C38, 05C45.

Here, all graphs are undirected, finite, connected, and neither contain loops nor multiple edges. Let $G$ be a graph. If a vertex of $G$ has degree 3, then we call the vertex cubic. If every vertex of $G$ is cubic, we call $G$ cubic. $G$ is hamiltonian if it contains a hamiltonian cycle, i.e. a cycle passing through all vertices of the graph. $G$ is hypohamiltonian if $G$ does not contain a hamiltonian cycle but for any vertex $v$ of $G$, the graph $G-v$ does contain a hamiltonian cycle. If we replace "cycle" by "path", we obtain the definition of a hypotraceable graph.

Let $n_{0}\left(c_{0}\right)$ be the smallest natural number such that there exists a planar (planar cubic) hypohamiltonian graph of order $n$ for every $n \geq n_{0}$ (for every even $n \geq c_{0}$ ).

The solution to the first problem we want to present would constitute an improvement to the main result from [4]. We consider it to be very interesting, since many consequences concerning problems on longest paths and cycles arise, but also very difficult. Similarly, we consider Problem 4 to be especially intriguing.

1. In [4], together with Jooyandeh, McKay, Östergård, and Pettersson, we showed that there exist 25 planar hypohamiltonian graphs of order 40 . Despite the progress made in [4], there still is a considerable gap between the order of the smallest known planar hypohamiltonian graph, which is 40 , and the best lower bound known for the order of the smallest such graphs, which is 18 , see [1].

(a) Do planar hypohamiltonian graphs on less than 40 vertices exist?

(b) What is $n_{0}$ ? (It is known that $n_{0} \leq 42$, see [4].)

*The author is a PhD fellow at Ghent University on the BOF (Special Research Fund) scholarship $01 \mathrm{DI} 1015$. 
2. Araya and Wiener showed that there exists a planar cubic hypohamiltonian graph on 70 vertices [2].

(a) Is there such a graph with fewer vertices?

(b) Correspondingly, we ask for $c_{0}$. (Presently, we know that $c_{0} \leq 74$, see [6].)

A graph $G$ is almost hypohamiltonian if $G$ is non-hamiltonian, and there exists a vertex $w$, which we will call exceptional, such that $G-w$ is non-hamiltonian, yet for any vertex $v \neq w$ the graph $G-v$ is hamiltonian. Define $n_{0}^{\prime}$ to be the smallest natural number such that there exists a planar almost hypohamiltonian graph of order $n$ for every $n \geq n_{0}^{\prime}$.

3. (a) We know that there exists an almost hypohamiltonian graph on 17 vertices [6]. Are there smaller examples?

(b) Do almost hypohamiltonian graphs of order $n \in\{18,19,21,24\}$ exist?

4. Thomassen's question from 1978 whether 4-connected hypohamiltonian graphs exist remains open [5]. We ask here:

(a) Do 4-connected hypotraceable graphs exist? (Horton [3] showed that 3-connected hypotraceable graphs exist.)

(b) Do 5-connected almost hypohamiltonian graphs exist? (We know that 4-connected almost hamiltonian graphs do exist [6].)

Note that, by results from [6], a positive answer to (a) would imply a positive answer to (b), but not necessarily vice-versa.

5. Is there an almost hypohamiltonian graph with a cubic exceptional vertex and all other vertices of degree at least 4? Solving this would answer, by using a result from [6], Thomassen's question whether hypohamiltonian graphs with minimum degree 4 exist [5].

6. (a) Is there a planar almost hypohamiltonian graph with fewer than 39 vertices? (An example with 39 vertices is available [6].)

(b) What is $n_{0}^{\prime}$ ? (So far, it is known that $n_{0}^{\prime} \leq 76$, see [6].)

7. Due to certain gluing results, planar almost hypohamiltonian graphs with cubic exceptional vertex are of special interest. In [6] it is shown that there exists a planar almost hypohamiltonian graph of order 47 whose exceptional vertex is cubic. Are there smaller examples? (This would help improve the bound for $n_{0}^{\prime}$.) 


\section{References}

[1] R. E. L. Aldred, B. D. McKay, and N. C. Wormald. Small Hypohamiltonian Graphs. J. Combin. Math. Combin. Comput. 23 (1997) 143-152.

[2] M. Araya and G. Wiener. On cubic planar hypohamiltonian and hypotraceable graphs. Electron. J. Combin. 18 (2011) \#P85.

[3] J. D. Horton. A Hypotraceable Graph. Research Report CORR 73-4, Dept. Combin. and Optim., Univ. Waterloo, 1973.

[4] M. Jooyandeh, B. D. McKay, P. R. J. Östergård, V. H. Pettersson, and C. T. Zamfirescu. Planar hypohamiltonian graphs on 40 vertices. Submitted.

[5] C. Thomassen. Hypohamiltonian graphs and digraphs. Theory and Applications of Graphs, Lecture Notes in Mathematics 642, Springer, Berlin (1978) 557-571.

[6] C. T. Zamfirescu. On hypohamiltonian and almost hypohamiltonian graphs. J. Graph Theory 79 (2015) 63-81.

Carol T. Zamfirescu

Department of Applied Mathematics, Computer Science and Statistics,

Ghent University, Krijgslaan 281 - S9, 9000 Ghent, Belgium.

czamfirescu@gmail.com 